\title{
Fitness networks for real world systems via modified preferential attachment
}

\author{
Ke-ke Shang \\ School of Marine Science and Technology, Northwestern Polytechnical University, Xi'an 710072, P. R. China \\ School of Mathematics and Statistics, The University of Western Australia, Crawley, WA, 6009, Australia \\ Michael Small \\ School of Mathematics and Statistics, The University of Western Australia, Crawley, Western Australia, 6009, \\ Australia \\ Mineral Resources, CSIRO, Kensington, Western Australia, 6151, Australia \\ Wei-sheng Yan \\ School of Marine Science and Technology, Northwestern Polytechnical University, Xi'an 710072, P. R. China
}

\begin{abstract}
Complex networks are virtually ubiquitous, and the Barabási and Albert model ( $B A$ model) has became an acknowledged standard for the modelling of these systems. The so-called $B A$ model is a kind of preferential attachment growth model based on the intuitive premise that popularity is attractive. However, preferential attachment alone is insufficient to describe the diversity of complex networks observed in the real world. In this paper we first use the accuracy of a link prediction method, as a metric for network fitness. The link prediction method predicts the occurrence of links consistent with preferential attachment, the performance of this link prediction scheme is then a natural measure of the "preferential-attachment-likeness" of a given network. We then propose several modification methods and modified $B A$ models to construct networks which more accurately describe the fitness properties of real networks. We find that all features assortativity, degree distribution and rich-club formation can play significant roles for the network construction and eventual structure. Moreover, link sparsity and the size of a network are key factors for network reconstruction. In addition, we find that the structure of the network which is limited by geographic location (nodes are embedded in a Euclidean space and connectivity is correlated with distances)
\end{abstract}

Email address: keke.shang@uwa.edu.au (Ke-ke Shang) 
differs from other typical networks. In social networks, we observe that the high school contact network has similar structure as the friends network and so we speculate that the contact behaviours can reflect real friendships.

Keywords: Complex network; $B A$ model; assortative; degree distribution; rich-club; network sparsity

\section{Introduction}

The Barabási and Albert preferential attachment growth model (the $B A$ model) [1] is the de facto standard model in the field of complex networks. The model is both intuitive and highly appealing. Popularity, as characterised by node degree, is a sufficient mechanism to lead to the

5 often treated as the generative process for a wide range of real scale-free network systems. However, the $B A$ model follows the simple principle that popularity is attractive [2] whereas the real world is often more complicated $[2,3,4]$. Similarity (or affinity) between nodes [2], minimum node degree [3] and the dominance of low degree nodes [4] all play significant supporting roles while constructing fitness networks ${ }^{1}$.

Emerging as the solution to a quite seperate problem, link prediction can help us to analyse and predict network structure in real-world networks with characteristic structure $[5,6,7]$. By taking the $B A$ model as the underlying generative pattern, we aim to adapt a newly described link prediction method $(P A I)[8]$ to predict links based on the degree to which preferential attachment is followed and hence measure the level of preferential attachment in real networks. Hence, we propose using the accuracy of the PAI algorithm to measure the fitness between these network construction models and real networks. Obviously, the BA model cannot model the degree distribution for many real networks. Conversely, the configuration model $[9,10]$ can achieve the same degree distribution as real networks, but it is difficult to maintain the connectivity of the networks and degree distribution and the connectivity as metrics of networks fitness.

In our previous studies $[3,4]$, we observed that the assortativity of a $B A$ network differs from

\footnotetext{
${ }^{1}$ In our paper, the fitness network means that a network has the similar structure, degree distribution and connectivity with the original real network.
} 
that of typical real networks. Furthermore, previous studies already proposed the assortative preferential attachment to construct the scale-free network with a given assortativity value [11] or can control their model parameter to change the network assortativity [12]. A specific modified preferential attachment also has been proposed to describe the local assortativity distribution for the Internet Autonomous System level networks [13]. In this paper, based on Xulvi-Brunet and Sokolov's algorithm [14], we use corresponding randomized methods to modify the assortativity of an unbiased $B A$ network to construct fitness networks - networks which better match real observed data.

However, we find that these assortativity modification methods are not successful in all cases. Moreover, since the actual degree distribution of $B A$ models will usually differ from that of real networks, we further propose two modified methods to modify the degree distribution and the product of nodes degrees of the $B A$ networks. Finally we note that in our previous study we found other side, our modified methods cannot work well for the the large size and the sparse network. Hence, to construct the fitness network simpler and more efficient, we propose one additional modified $B A$ model to construct networks with more rich-clubs and one additional modified $B A$ model to construct networks with more randomly links. In addition, compare these two additional which more effectively construct fitness networks.

In this paper, we use 14 different real datasets to construct a range of archetypal "real" networks. We use the $B A$ model to construct corresponding networks. Meanwhile, we compute the connectivity, the accuracy of $P A I$ and the degree distribution as metrics of network fitness. Our various modified methods (which will be described below) and network generation models all maintain the connectivity of the original real networks. However, compared to real networks, we find that there is only one $B A$ network which matches according to the second metric. Hence, we use our methods to modify the assortativity of the $B A$ networks, after which, we find that there are now six modified $B A$ networks coincide according to the second metric. Finally, we use our new methods to modify the degree distribution and the product of nodes degrees of $B A$ networks, following this we find that there 12 networks are able to match the model with the second metric. Based on the rich-club phenomena and random networks, we propose two network models $R C B A$ and $R B A$ respectively. Moreover, we propose the corresponding modified models of $R C B A$ 
$(M R C B A)$ and $R B A(M R B A)$. Finally, we can combine our modified methods and models to more similar to the original real networks. In addition, we find that the human behaviours can reflect underlying real ("true") friendships. The network which is limited by the geographic location has the special structure, and the rich-club, the sparsity and the size of network all play the key roles for the network reconstruction.

\section{Data}

Different kinds of networks have different structures for the reconstruction, to consider the diversity of networks, we employ large open and publically available data sets from 14 different undirected networks: from the social network to the engineering network, from the sparse network to the dense network, from the small size network to the large size network. (1) Football network football player which participated in the World Championship in Paris, 1998. (2) Dolphin network [17]: a node represents one dolphin which in a community living off Doubtful Sound, a link stands for there is associations between a pair of nodes. (3) PolBooks network [16]: a node represents one book about US politics which is sold by Amazon.com, a link stands for there is a pair of nodes student, a link stands for one node reported a friendship with another one. We get link information from the publically site SocioPatterns directly [19]. (5) C.Elegans network [20]: a node represents one neuron, a link indicates a synapse or a gap junction between a pair of nodes. (6) Contact $_{1}$ network [18]: a node represents one high school student, a link indicates the face to face contact of a pair of nodes. The students of the Friendship network also are the students of the Contact $_{1}$ network. We get link information from the publically site SocioPatterns directly [19]. (7) USAir network [16]: a node represents one airport, a link indicates there is a flight line between a pair of nodes. (8) SmaGri network [16]: a node represents one author who cite the papers of Small \& Griffith and Descendants, a link indicates a pair of nodes who cooperate on a paper. (9) Pblog network [21]: a node represents one blog on US politics, a link indicates a pair of nodes have hyperlinks between them. (10) NetScience network [22]: a node represents one author working on network science, a link indicates there is a co-authorship between a pair of nodes. (11) Yeast network [23]: a node represents one protein in budding yeast, a link indicates there is interactions 
between a pair of nodes. (12) USPower network [20]: a node represents one power station of the Western states of US, a link indicates there is electric cables between a pair of nodes. (13) Contact $_{2}$ network [24]: a node represents one person in the infectious social networks, a link stands for the contact of a pair of nodes. We get link information from the publically site SocioPatterns directly [19]. (14) Router network [25]: a node represents one router at the level of autonomous systems, a link indicates the traffic flow between a pair of nodes. Table. 1 provides the number of nodes and links for each network.

Table 1: The number of nodes $(N)$ and links $(L)$, and the ratio of the $L$ to the $N$ for 14 networks. We do not count the number of loop links, the isolated nodes and links, and the duplicate nodes and links. Here, $L / N$ can reflect the sparsity of the network. The networks which have the lower value of $L / N$ are sparse networks, such as the Netscience network, the USPower network and the Router network.

\begin{tabular}{|c|c|c|c|c|c|c|c|}
\hline & Football & Dolphin & PolBooks & Friendship & C.Elegans & Contact $_{1}$ & USAir \\
\hline$N$ & 35 & 63 & 105 & 134 & 296 & 327 & 332 \\
\hline$L$ & 118 & 159 & 441 & 406 & 2148 & 5818 & 2126 \\
\hline \multirow[t]{2}{*}{$L / N$} & 3.3714 & 2.5238 & 4.2000 & 3.0298 & 7.5267 & 17.7920 & 6.4036 \\
\hline & SmaGri & Pblog & NetScience & Yeast & USPower & Contact $_{2}$ & Router \\
\hline$N$ & 1059 & 1222 & 1589 & 2361 & 4941 & 10972 & 22963 \\
\hline$L$ & 4916 & 16714 & 2742 & 6646 & 6594 & 44517 & 48436 \\
\hline$L / N$ & 4.6421 & 13.2357 & 1.7256 & 2.8149 & 1.4074 & 4.0573 & 2.1093 \\
\hline
\end{tabular}

\section{Metrics and methods}

In this section we introduce the metrics which we will employ to assess the fitness network generation models and the similarity to real data. The metrics described below measure several aspects of the networks: connectivity; accuracy of $P A I$, or faithfulness of the network to that expected from preferential attachment; and, degree distribution. We then describe the standard link prediction problem: the definition of link prediction; the link prediction method PAI; and, how to measure the accuracy of $P A I$. Finally, we will describe the process via which we are able to construct fitness networks. To do this we will propose two modification methodologies to alter the structure of networks generated via existing methods and four distinct network models to attempt to close the gap between the output of the $B A$ model, and most real world data. 


\subsection{Metrics}

In previous studies, link prediction methods have been applied to analyse the structure of networks $[5,6,7]$. The $B A$ model is the foundational method for the construction of complex networks $[3,4]$ and has been widely used and widely discussed. The seperate preferential attachment index methods $(P A I)[8]$ for link prediction are based on the premise that the $B A$ model [1] can reflect the level of preferential attachment and the structural properties of the networks. Hence, we take the connectivity, the accuracy of $P A I$, the degree distribution as the metric to measure the similarities of our models with original real networks or the construction of fitness networks.

Meanwhile, the standard link prediction problem, as considered by [26], can be formulated

as follows. From the non-observed links construct the set $\bar{E}$, the observed link set $E$ is divided into two parts $E^{T}$ and $E^{P}$ where $E^{T} \cup E^{P}=E$ and $E^{T} \cap E^{P}=\emptyset$. The division into $E^{P}$ (typically including $10 \%$ of the observed links in [26]) and $E^{T}$ (typically $90 \%$ of the observed links) is arbitrary and will be used for scoring purposes. That is, all the links in $E=E^{P} \cup E^{T}$ have been observed and are known, however, links in $E^{T}$ will form a training set and are used to implement a link prediction score, the efficacy of which will be evaluated over the probe set $E^{P}$. In addition, the $P A I$ can be simply described as follows: $S$ core $_{x y}=k_{x} \times k_{y}$, where $k_{i}$ is the degree of node $i$. The so-called receiver operating characteristic curve $(A U C)$, originally applied to evaluate communication schemes, has since been widely applied to measure prediction accuracy [27] in a variety of settings. Here, we use $A U C$ as a prediction accuracy measure for networks. Only the information of $E^{T}$ is allowed to be used to compute the performance score $S c o r e_{x y}$, we compare the prediction scores of $m$ pairs of nodes from $E^{P}$ and $\bar{E}$ randomly, if there are $m^{\prime}$ times that the score measured from $E^{P}$ is bigger than the score measured from $\bar{E}$ and $m^{\prime \prime}$ times that the two scores are equal, then, $A U C=\left(m^{\prime}+0.5 m^{\prime \prime}\right) / m$. Here, the $A U C$ value is closer to 1 means that the link prediction method is more efficient. Moreover, the $A U C$ values are determined by the relationship between the network structure and the link prediction algorithm.

As shown in Fig. 1, we describe a simple example to explain the $A U C$ schematic. Here, we only compute the $A U C$ once $(m=1)$ : First, the observed link set (a) can be divided into a training set (b) and a probe set (c). Next, we can choose a pair of nodes $A B$ from the probe set (c) and a pair of nodes from the non-observed links set (d). Only the training set (b) can be used to compute the node degree. If we choose the pair of nodes $A D$ from the non-observed links set (d), Score $_{A B}=k_{A} \times k_{B}=0$, Score $_{A D}=k_{A} \times k_{D}=1$, namely Score $_{A B}<$ Score $_{A D}$, 
hence $m^{\prime}=0, m^{\prime \prime}=0$, then $A U C=(0+0.5 \times 0) / 1=0$. If we choose the pair of nodes $B D$ from the non-observed links set (d), Score $_{A B}=k_{A} \times k_{B}=0$, Score $_{B D}=k_{B} \times k_{D}=0$, namely Score $_{A B}=$ Score $_{B D}$, hence $m^{\prime}=0, m^{\prime \prime}=1$, then $A U C=(0+0.5 \times 1) / 1=0.5$.

(b)

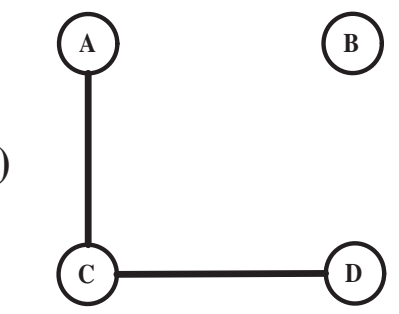

\section{Training set}

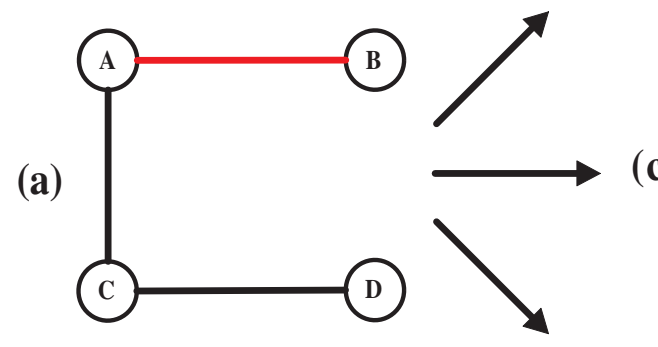

(c)

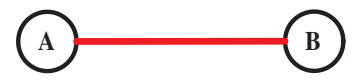

Probe set

\section{Observed links}

(d)

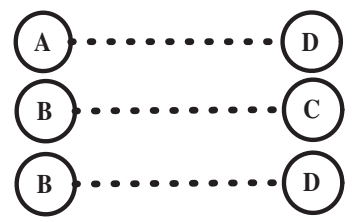

Non-observed links

Figure 1: (Color online) The simple examples of the observed links set $E$, the non-observed links set $\bar{E}$, the training set $E^{T}$ and the probe set $E^{P}$.

Not all real networks, built via growth process and exhibiting scale-free characteristics, share the statistical features of the $B A$ model. We have previously observed that both assortativity $[3,4]$ and the degree distribution of $B A$ networks differ from that observed in real networks. Moreover, the rich-club plays a significant roles for other properties of networks [15], we propose two modification 
methods and four network models to modify networks generated via the $B A$ process and construct fitness networks.

\subsubsection{Modification methods}

First, we can use randomized methods to modify the structure of $B A$ networks. As shown in Fig. 2, we use the randomized edges method $(R E)[28]$ to change the structure, the assortativity and the rich-club properties of $B A$ networks [29], but the degree distribution of every $B A$ network is maintained.

\section{$\mathbf{R} \mathbf{E}$}

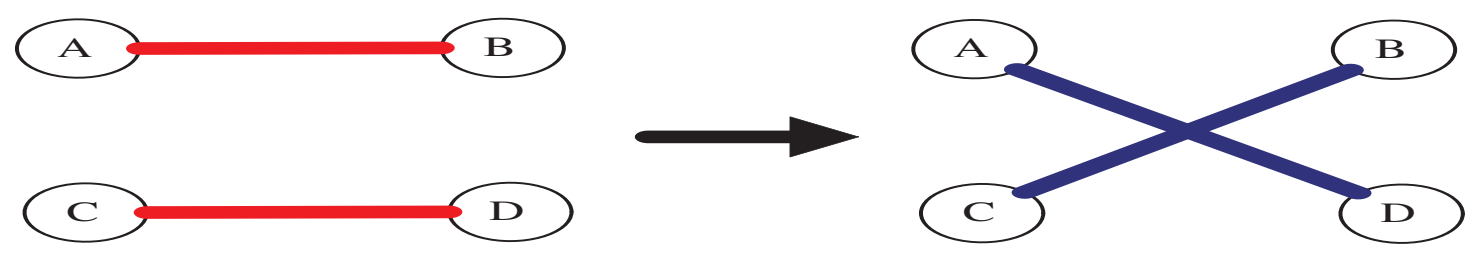

Figure 2: (Color online) Nodes $A$ and $B$, and nodes $C$ and $D$ are connected, while nodes $A$ and $D$, and nodes $B$ and $C$ are not connected. Then cut the links $A B$ and $C D$, and connect the nodes $A$ and $D$, the nodes $B$ and $C$ respectively.

In our previous study, we found that the assortativity of real networks differs from that of $B A$ networks $[3,4]$ and we also observed that we can change the assortativity of these original networks by controlled application of $R E$ [30]. Based on Xulvi-Brunet and Sokolov's algorithm [14], as shown in Fig. 3, we use the disassortative $R E$ method $(D A R E)$ and the assortative $R E$ method $(A R E)$ to change the structure and the assortativity of the original $B A$ networks. In each network, there is a limited number of motifs which are described in Fig. 3, but we simply continue the randomization process until convergence.

In addition we can aim to directly improve the degree product of every pair of nodes which have a link between them to improve the accuracy of $P A I$. Conversely, we could decrease the degree product of every pair of nodes which have a link between each other to decrease the performance 
(a)

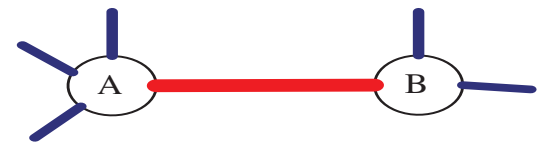

\section{A R E}
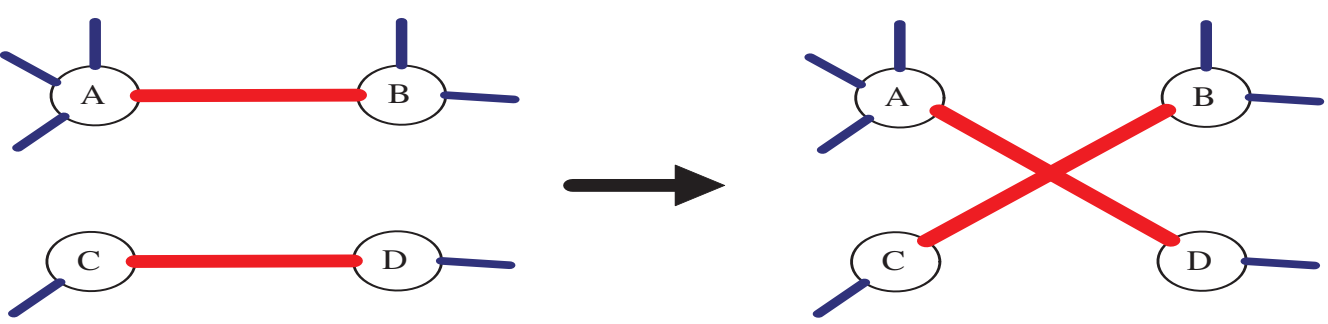

(b)

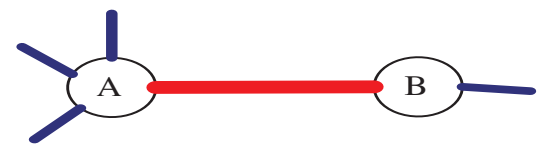

A RE
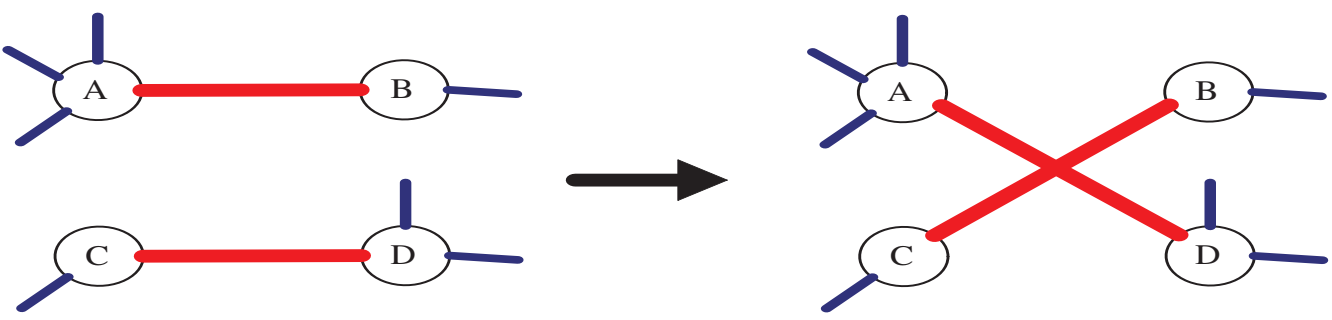

Figure 3: (Color online) (a) Nodes $A$ and $B$, and nodes $C$ and $D$ are connected, while nodes $A$ and $D$, and nodes $B$ and $C$ are not connected. In addition, $\left|k_{A}-k_{B}\right|<\left|k_{A}-k_{D}\right|$ and $\left|k_{C}-k_{D}\right| \leq\left|k_{C}-k_{B}\right|$, where $k_{i}$ is the degree of node $i$. Then cut the links $A B$ and $C D$, and connect the nodes $A$ and $D$, the nodes $B$ and $C$ respectively. (b) Nodes $A$ and $B$, and nodes $C$ and $D$ are connected, while nodes $A$ and $D$, and nodes $B$ and $C$ are not connected. In addition, $\left|k_{A}-k_{B}\right|>\left|k_{A}-k_{D}\right|$ and $\left|k_{C}-k_{D}\right| \geq\left|k_{C}-k_{B}\right|$, where $k_{i}$ is the degree of node $i$. Then cut the links $A B$ and $C D$, and connect the nodes $A$ and $D$, the nodes $B$ and $C$ respectively.

of $P A I^{2}$. Doing so, we obtain similar performance ${ }^{3}$, similar degree distribution and the same connectivity as the original networks. The process is depicted in Fig. 4. We propose two new randomization methods to change the structure and the degree distribution of $B A$ networks. These two methods not only can change the degree distribution of $B A$ networks, but also can change the

\footnotetext{
${ }^{2}$ The score of $P A I$ is determined by the degree product of every pair of nodes. On the other side, $A U C$ compare the score of the pair of nodes which have a link between each other and that have no link between each other. Hence, if we decrease or increase the degree product of every pair of nodes which have a link, we can decrease or increase the performance of $P A I$ respectively.

${ }^{3}$ Here, we define the similar performance as the difference between the $A U C$ values of our models and that of original networks are not larger than 0.02 .
} 
PAI performance of networks. Moreover, because the pair of nodes which links will be cut have the common neighbours, hence our randomized methods can maintain the connectivity of networks. We denote these methods as increased $R E$ method (IRE) and decreased $R E$ method ( $D R E)$. As before, while in each network, there is a limited number of motifs which are described in Fig. 4, we will randomize until convergence.

(a)

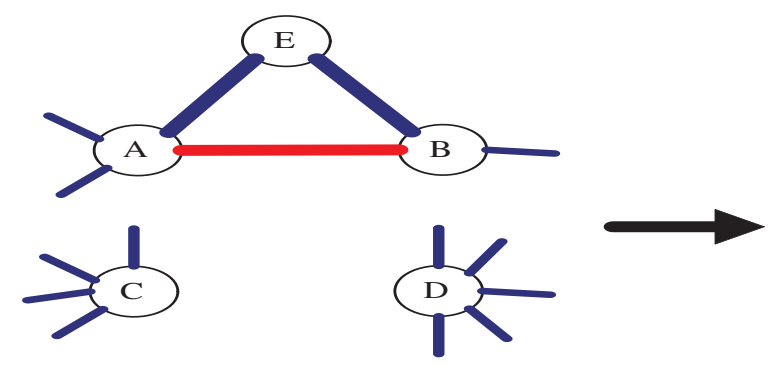

(b)

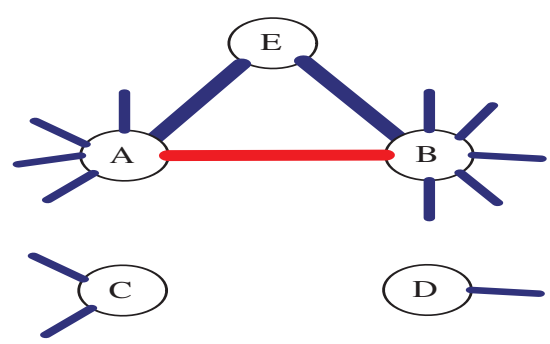

\section{R E}

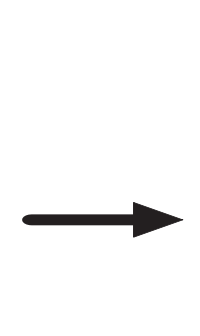

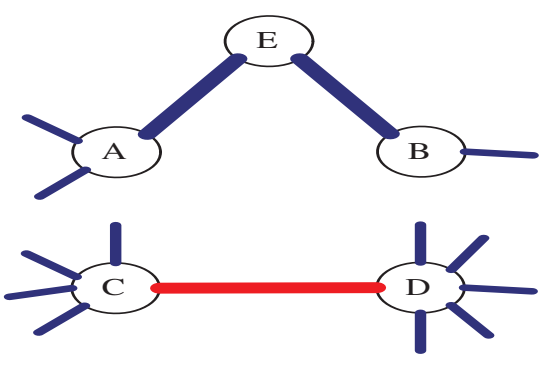

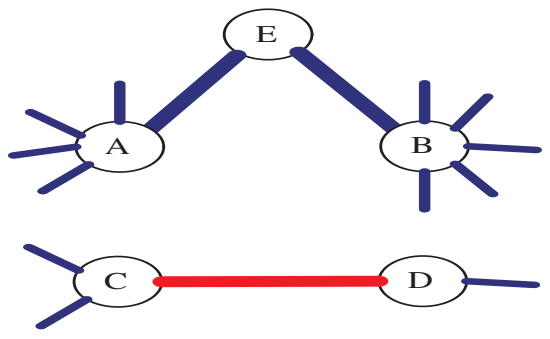

Figure 4: (Color online) (a) Nodes $A$ and $B$ are connected, and should have a common neighbour $E$, while nodes $C$ and $D$ are not connected. In addition, $k_{A} \times k_{B}<k_{C} \times k_{D}$, where $k_{i}$ is the degree of node $i$. Then cut the link $A B$ and connect the nodes $C$ and $D$. (b) Nodes $A$ and $B$ are connected, and should have a common neighbour $E$, while nodes $C$ and $D$ are not connected. In addition, $k_{A} \times k_{B}>k_{C} \times k_{D}$, where $k_{i}$ is the degree of node $i$. Then cut the link $A B$ and connect the nodes $C$ and $D$.

\subsubsection{Network models}

In our previous studies, we found that the rich-club plays a significant role on the other structural properties of networks [15]. Hence, we also propose two modified $B A$ models targetting these rich-clubs.

First, we propose a new model which follows the rich-club phnomenoa and has the same number 
of nodes and links as the original network. If the original real network has $m+t$ nodes and $N$ links: (1) Given $m(m \geqq 1)$ nodes, construct a fully-connected network. (2) Next, introduce one new node to only connect to one existing node every time, and the connected probability of existing node $i$ (degree $k_{i}$ ) is $P_{i}=\frac{k_{i}}{\sum_{j=1}^{m+t} k_{j}}$. After $t$ times, we could construct the network which have $m+t$ nodes and $m(m-1) / 2+t$ links. (3) Finally, we add $N-m(m-1) / 2-t$ links to the pairs of nodes which have no link between them, and the connected probabilities of existing nodes both are equal to that of step (2), which means both the two connected nodes are more like rich nodes. We named this model as the rich-club $B A$ model $(R C B A)$.

Second, we propose a new model which (as above) we first build a tree via preferential attachment and then (different from RCBA) adds additional links randomly - until one has the same number of nodes and links as the original network. If the original real network has $m+t$ nodes and $N$ links. (1) Given $m(m \geqq 1)$ nodes, construct a fully-connected network. (2) Next, introduce one new node to only connect to 1 existing node every time, and the connected probability of existing node $i$ (degree $k_{i}$ ) is $P_{i}=\frac{k_{i}}{\sum_{j=1}^{m+t} k_{j}}$. After $t$ times, we could construct the network which have $m+t$ nodes and $m(m-1) / 2+t$ links. (3) Finally, we add $N-m(m-1) / 2-t$ links randomly. We named this model as the random $B A$ model $(R B A)$.

In addition, for the step (2) of the $R C B A$ and the $R B A$, we can use the method as shown in Fig. 5 to exchange the new added link. For step (3) of the $R C B A$ and the $R B A$, we can use the method as shown in Fig. 6 to exchange the newly added links. We named the modified models as $M R C B A$ and $M R B A$. We summarise the various algorithms describe above in Table. 2.
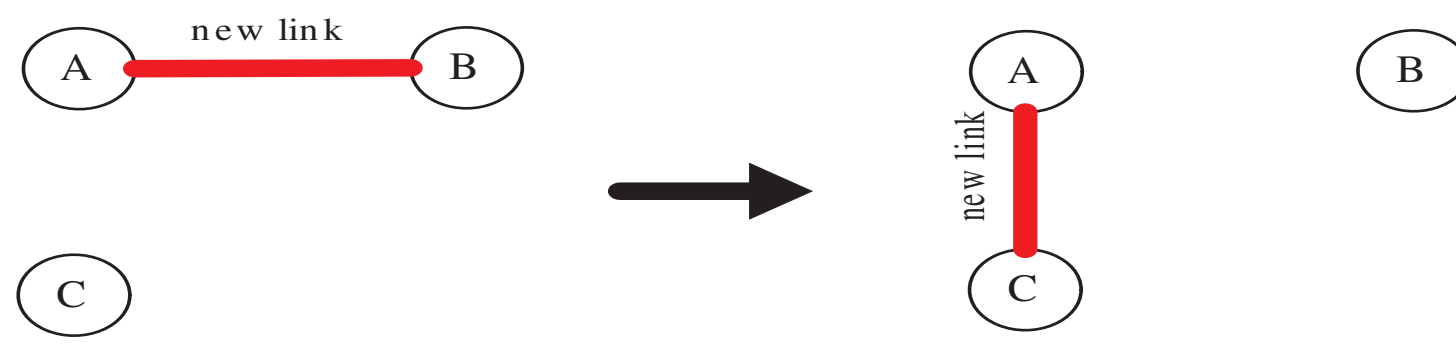

Figure 5: (Color online) Link $A B$ is the new added link, node $A$ is the new node, then cut the link $A B$ and connect the nodes $A$ and $C$. If the $P A I$ performance of our networks are lower than that of original real networks, then $k_{C}>k_{B}$. If the $P A I$ performance of our networks are better than that of original real networks, then $k_{C}<k_{B}$. 


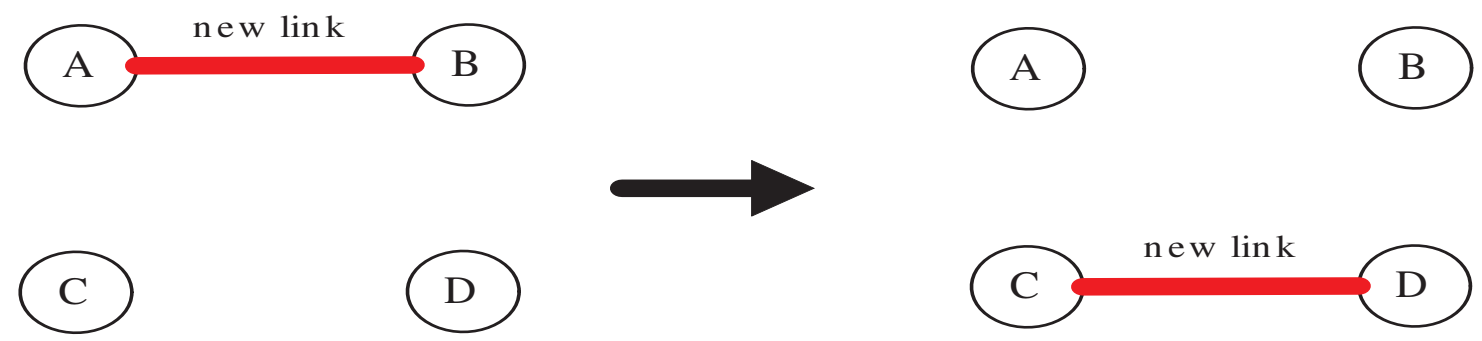

Figure 6: (Color online) Link $A B$ is the new added link, nodes $C$ and $D$ are not connected, then cut the link $A B$ and connect the nodes $C$ and $D$. If the $P A I$ performance of our networks are lower than that of original real networks, then $k_{C} \times k_{D}>k_{A} \times k_{B}$. If the $P A I$ performance of our networks are better than that of original real networks, then $k_{C} \times k_{D}<k_{A} \times k_{B}$.

Table 2: All our methods which are described in Section. 3.2. Here, we describe all the purposes of our methods. For all our methods, the connectivity of original real networks is maintained.

\begin{tabular}{cc}
\hline \hline Methods & Description \\
\hline$R E$ & Change the structure, the assortativity and the rich-club properties of networks (see Fig. 2). \\
$D A R E$ & Change the structure and increase the disassortativity of networks (see Fig. $3(\mathrm{a})$ ). \\
$\begin{array}{c}A R E \\
I R E\end{array}$ & Change the structure and increase the assortativity of networks (see Fig. 3 (b)). \\
$D R E$ & Change the structure and increase the $P A I$ performance of networks (see Fig. $4(\mathrm{a}))$. \\
$R C B A$ Model & Change the structure and decrease the $P A I$ performance of networks (see Fig. $4(\mathrm{~b}))$. \\
$R B A$ Model & The modified $B A$ model which can construct more rich-clubs. \\
$M R C B A$ Model & The modified $B A$ model which is more like random networks. \\
$M R B A$ Model & The modified $R B A$ model which either can increase or decrease the $P A I$ performance. \\
\hline \hline
\end{tabular}

\section{Results}

We now present the results of computations. We examine the similarity between the various $B A$ models ( $B A$ model and modified $B A$ models) and the real networks by our methods. We find that the $B A$ model only matches one of our real world networks. The modified $B A$ model matches 12. Modifications applied to our generative models can match all 14 fitness networks.

\subsection{BA model}

To build the corresponding complex networks which have the same number of nodes and the similar number of links as the original networks, we use the modified $B A$ model to construct the fully-connected networks [1]: Given $m(m \geqq 2)$ nodes, construct a fully-connected network. Next, introduce one new node to connect to $n$ or $n+1(1 \leqslant n<n+1 \leqslant m)$ existing nodes every time, and 
the connected probability of existing node $i$ (degree $k_{i}$ ) is $P_{i}=\frac{k_{i}}{\sum_{j=1}^{m+t} k_{j}}$. After $t$ times, we could construct our destination network which have $m+t$ nodes, the floating number of links is from $m(m-1) / 2+n t$ to $m(m-1) / 2+(n+1) t$. Finally, the probability of the degree $(k)$ distribution also follows a power-law distribution, $P_{k} \propto k^{-\gamma}$.

As shown in Fig. 7 , we find that the $P A I$ performance of $B A$ networks differ with that of original real networks except the Pbook network ${ }^{4}$. To get the similar results of original networks, we try to use randomized methods to modify $B A$ networks. Hence, we use $R E$ to change the structure, the assortativity and the rich-club properties of $B A$ networks. However, as shown in Fig. 7, we see that the $P A I$ performance of modified $B A$ networks which are randomized by $R E$ have no big differences with that of $B A$ networks. Here, the number of $R E$ randomizations for each network is equal to the 10 times of the number of links. Interestingly, we find that the $P A I$ performance of the original contact network and the original friendship network which are from same high school are similar to each other. We suggest that human behaviours can reflect their real friendships to some extent.

We find that the assortativity of real network differ with that of $B A$ networks $[3,4]$ and we are able to change the assortativity of original networks by controlled application of $R E$ [30]. Here, we use our new methods DARE and $A R E$ to change the structure and the assortativity of original $B A$ networks. As shown in Fig. 7 , we see that the $P A I$ performance of $B A$ networks which are randomized by $D A R E$ are better than that of $B A$ networks, and not lower than that of $B A$ networks which are randomized by ARE except for the USPower network. However, we can find that the $P A I$ performance of four original networks are located between that of the modified $B A$ networks which are randomized by $D A R E$ and that of the modified $B A$ networks which are randomized by $A R E$. The modified NetScience and Contact $_{1} B A$ networks can get the similar PAI performance as the original real network. These phenomena suggest that only six different $B A$ networks which are randomized by $D A R E$ or $A R E$ can achieve the similar performance as corresponding original networks. Specially, differ from other networks, we find that the performance of modified USpower $B A$ network by DARE are lower than that of modified USpower $B A$ networks by $A R E$, due to only the power network is limited to the geographic location. However, we can not find a similar

\footnotetext{
${ }^{4}$ Only by computing the $A U C$ accuracy of $P A I$ about 10, 000 times, we can consistently achieve the stable results. Hence, we compute the $A U C$ accuracy of $P A I$ more than 10,000 times independently.
} 


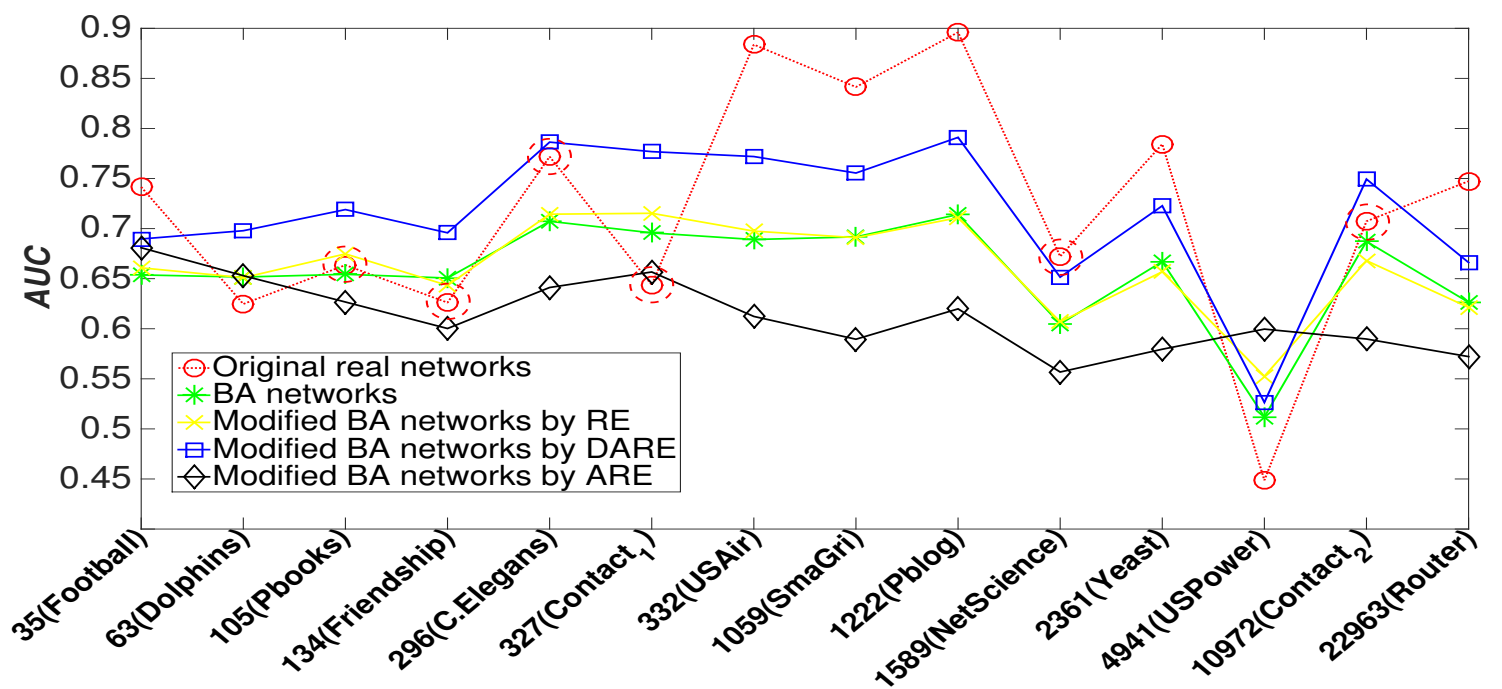

Figure 7: (Color online) Under the metric of the $A U C$ accuracy of $P A I$, the performance of original real networks, $B A$ networks and modified $B A$ networks. The circles with dashed lines of the original real networks mean that we can use our methods to archive the similar $P A I$ performance of the original networks.

phenomena in contact networks, due to the fact that the contact networks actually are offline social networks which are not limited by the geographic location in a traffic and communication developed society. On the other side, these phenomena suggest that we can not use DARE and ARE to modify the USPower BA network - interestingly this is a spatially engineered network, it is deliberately designed rather than the evolved network. Table. 3 provides the number of randomizations for each network.

Table 3: The number of randomizations for (DARE and $A R E)$ of each network for the results which shown in Fig. 7.

\begin{tabular}{cccccccc}
\hline \hline & Football & Dolphin & PolBooks & Friendship & C.Elegans & Contact $_{1}$ & USAir \\
\hline DARE & 42 & 49 & 178 & 131 & 1045 & 3243 & 1125 \\
ARE & 61 & 96 & 341 & 372 & 2410 & 6689 & 2878 \\
\hline & SmaGri & Pblog & NetScience & Yeast & USPower & Contact $_{2}$ & Router $^{\text {Netent }}$ \\
\hline DARE & 2376 & 9092 & 1033 & 2614 & 1125 & 18555 & 16312 \\
ARE & 7314 & 26493 & 3891 & 9296 & 2878 & 66416 & 67383 \\
\hline \hline
\end{tabular}

Furthermore, as shown in Fig. 11 (see Appendix), we find that the degree distributions of the $B A$ networks are different from those of the original networks. To get similar $P A I$ performance, more similar degree distribution and the same connectivity as the original networks, we use our 
new methods $I R E$ and $D R E$ to modify the $B A$ networks. As shown in Fig. 8 , we can see that the $P A I$ performance of the 10 different original networks are located between that of the modified $B A$ networks when randomized by $I R E$ and that of the modified $B A$ networks when randomized by $D R E$. These phenomena suggest that based on $B A$ model, we can use $I R E$ and $D R E$ to get the similar $P A I$ performance as 10 smaller size real networks. In addition, we see that the modified $B A$ network when randomized by $I R E$ also can get the similar performance as the real contact $_{2}$ network. However, compared to the Yeast $B A$ network and the contact $_{2} B A$ network, we find that the smaller size $B A$ network (NetScience) does not get the similar result as the original real network. Furthermore, we find that the NetScience network is more sparse than the Yeast network and the contact $_{2}$ network. Hence, we suggest that the sparsity of networks is one of the keys for the reconstruction of original networks. On the other side, compared to the $\operatorname{contact}_{2} B A$ network, we find that $I R E$ and $D R E$ are more effective for the smaller networks which have the similar sparsity as it. Hence, we suggest that the sparsity and the size both are keys for the reconstruction of original networks.

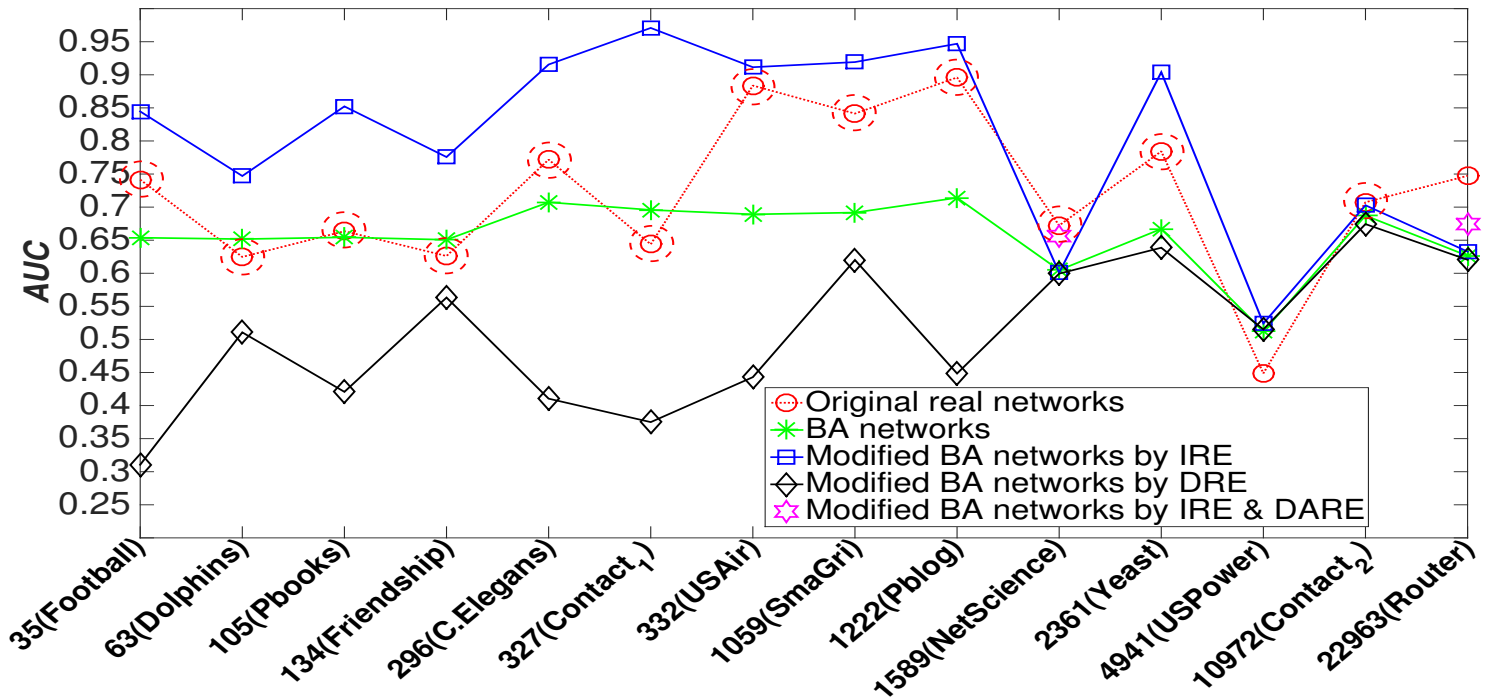

Figure 8: (Color online) Under the metric of the $A U C$ accuracy of $P A I$, the performance of original real networks, $B A$ networks and modified $B A$ networks.

Next, as shown in Fig. 8, we attempt to use $D A R E$ to modify the modified $B A$ networks (by $I R E)$ which do not achieve similar performances as the original networks, we can see that only the smaller size $B A$ network (NetScience) can get the similar performance as the original real network. 
This phenomenon also shows that the network size is one of the keys for the reconstruction of the original networks. Finally, we suggest that we can combine the product modified methods (IRE and $D R E$ ) and the assortativity modification methods ( $D A R E$ and $A R E$ ) to get more similar structures to the real networks. Table. 4 provides the number of randomizations for each network.

Table 4: The number of randomizations (IRE, DRE and DARE) for each network for the results which shown in Fig. 8.

\begin{tabular}{|c|c|c|c|c|c|c|c|}
\hline & Football & Dolphin & PolBooks & Friendship & C.Elegans & Contact $_{1}$ & USAir \\
\hline$I R E$ & 129 & 227 & 780 & 784 & 4178 & 11305 & 4592 \\
\hline$D R E$ & 407 & 50 & 241 & 98 & 1339 & 11306 & 1260 \\
\hline \multirow[t]{2}{*}{$D A R E$} & 0 & 0 & 0 & 0 & 0 & 0 & 0 \\
\hline & SmaGri & Pblog & NetScience & Yeast & USPower & Contact $_{2}$ & Router \\
\hline$I R E$ & 10560 & 33048 & 65 & 28654 & 10 & 1084 & 94 \\
\hline$D R E$ & 741 & 9706 & 52 & 281 & 44 & 1063 & 188 \\
\hline$D A R E$ & 0 & 0 & 1075 & 0 & 0 & 0 & 16356 \\
\hline
\end{tabular}

\subsection{New models and the role of rich-club}

Based on the $B A$ model, the modified methods cannot build all fitness networks for real data, and it is difficult for the configuration model to keep the connectivity of real networks. Especially for the large size network (Router network) and the sparse network (USPower network), the modified methods are not effective. Hence, to seek better models and study the role of the rich-club, we use our new models $R C B A$ and $R B A$ to construct the full-connected networks fitting real networks, then compare the performance of the two methods. As shown in Fig. 9, compared to $R B A$, we can see that the $P A I$ performance of the networks which are constructed by $R C B A$ are more similar to that of all original real networks except the USPower network. We can conclude that the rich-club plays a significant role for the construction of fitness networks.

Next, to obtain similar performance as the original networks, we try to combine the product modified methods ( $I R E$ and $D R E$ ) and the assortativity modification method ( $D A R E$ ) to modify our model. As shown in Fig. 10, we can see that the $P A I$ performance of about 12 different original networks are located between that of the modified $R C B A$ networks which are randomized by $I R E$ (or combined with $D A R E^{5}$ ) and that of the modified $R C B A$ networks which are randomized by

\footnotetext{
${ }^{5}$ Here, for the NetScience network, the number of $D A R E$ randomizations for $R C B A$ model is 1303 .
} 


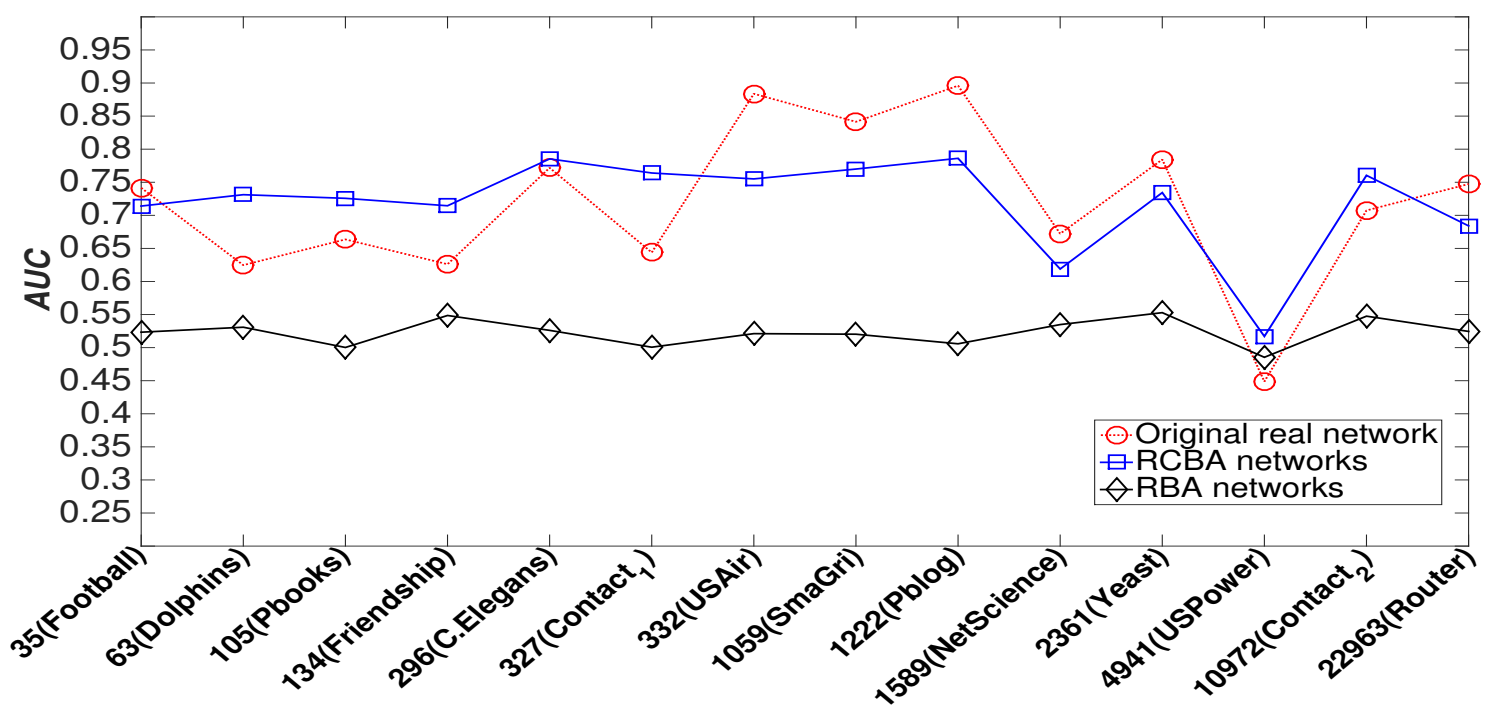

Figure 9: (Color online) Under the metric of the $A U C$ accuracy of $P A I$, the performance of original real networks, $R C B A$ networks and $R B A$ networks.

$D R E$. Hence, we suggest that we can modify the $R C B A$ networks to get the similar performance of all original real networks except the USPower network and the Router network. Table. 5 provides the number of randomizations for each network.

Table 5: The number of randomizations for each network for the results which shown in Fig. 10.

\begin{tabular}{cccccccc}
\hline \hline & Football & Dolphin & PolBooks & Friendship & C.Elegans & Contact $_{1}$ & USAir \\
\hline IRE & 107 & 176 & 738 & 756 & 4296 & 11194 & 4252 \\
DRE & 236 & 83 & 315 & 162 & 1856 & 11636 & 1273 \\
\hline \hline & SmaGri & Pblog & NetScience & Yeast & USPower & Contact $_{2}$ & Router $^{2}$ \\
\hline IRE & 9832 & 33428 & 141 & 24321 & 5 & 1791 & 95 \\
$D R E$ & 1166 & 14182 & 80 & 517 & 43 & 2410 & 77 \\
\hline \hline
\end{tabular}

Furthermore, we suggest that we can modify the $B A$ networks at every growth step. Hence, we use our new models $M R C B A$ and $M R B A$ to construct the full-connected fitness networks for the real world. As shown in Fig. 10, we use the $M R C B A$ model to construct the router network, and modified it by DARE (19436 times), then we can get the similar performance as the original router network. However, the MRCBA model does not work for the USPower network.

Moreover, under the metric of $A U C$, the $P A I$ performance of the original real network, $R B A$ 


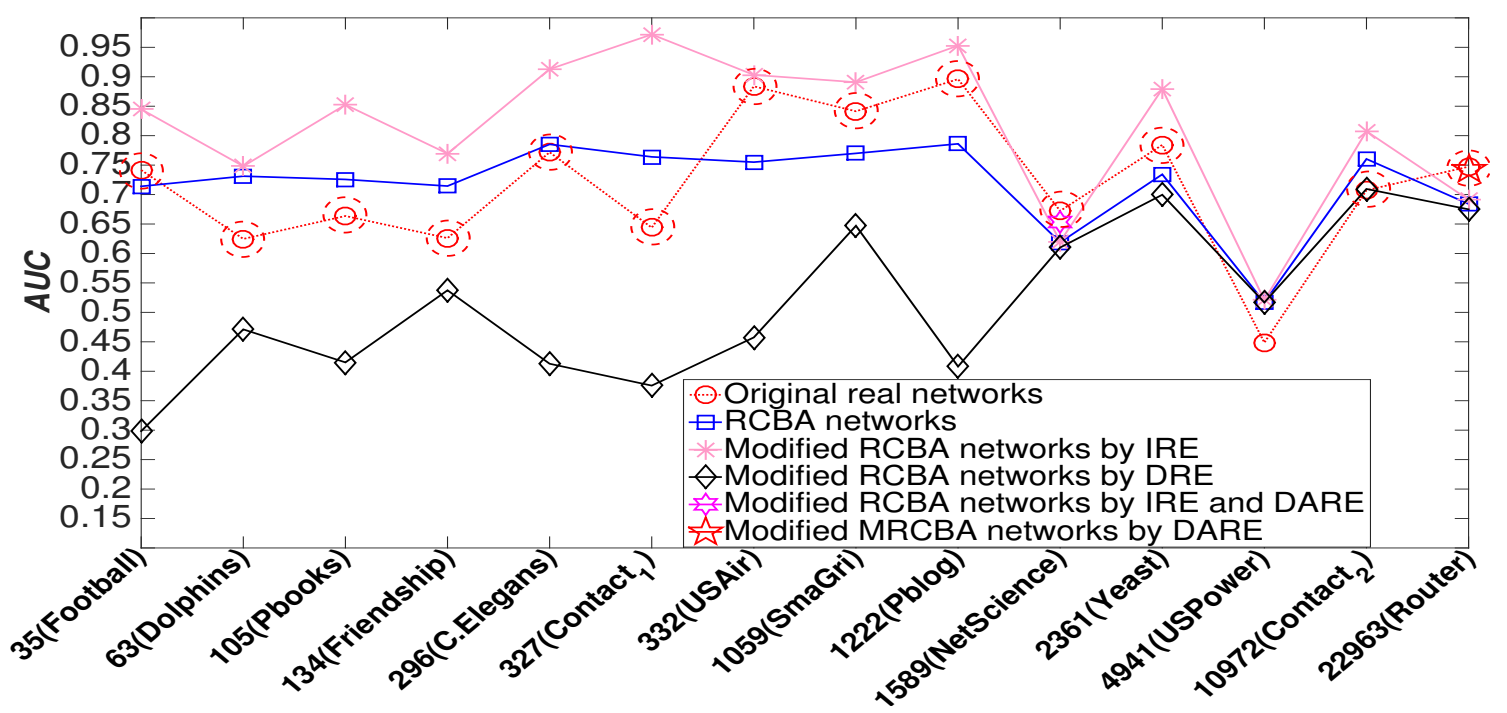

Figure 10: (Color online) Under the metric of the $A U C$ accuracy of $P A I$, the performance of original real networks, $R C B A$ networks, modified $R C B A$ networks and one modified $M R C B A$ network.

network and $M R B A$ network are $0.4487,0.4704$ and 0.3857 respectively. The $P A I$ performance of original real network is located between that of $R B A$ network and that of $M R B A$ network. We suggest that only the random model can get the similar structure of original USPower network. In addition, combine the special phenomena of USpower network as shown in Fig. 7 to Fig. 10, we can conclude that the structure of the network which is limited by the geographic location differs from that of other networks which are not limited by the geographic location, and is more like that of random networks.

In addition, as shown in Fig. 11 (see Appendix), compared to $B A$ networks, we find that the degree distributions of $R C B A$ networks are generally more similar to original real networks. Moreover, as shown in Fig. 12 (see Appendix) we use the USAir network as an example, describe the structure of original real network, $B A$ network and modified $B A$ network by IRE. We can see that the structure of the modified $B A$ network is more like the original real network. These phenomena also suggest that we can construct networks more similar to original real networks by our methods. 


\section{Discussion and conclusion}

In summary, we can use our new modified methods and models to construct the fitness networks for real world under our metrics. In detail, based on $B A$ model, we can combine our modified methods ( $D A R E$ and $I R E$, or $A R E$ and $D R E$ ) easily to construct the smaller size (the number of nodes is lesser than about 1500) or the dense $(L / N$ is bigger than about 2$)$ fitness networks. Furthermore, based on our rich-club model ( $R C B A$ and $M R C B A$ ), we can combine our modified methods to construct all fitness networks except the power network. In particular, we only can use the random network model to construct the fitness network for USPower network.

On the other hand, we find that the rich-club, the network sparsity and the network size all play important roles for the construction of fitness networks. Specially, we find that the power network is different with other networks and more like the random network, due to the role of the geographic location. We also find that the connect network actually is a offline social network and not limited by the geographic location in a traffic and communication developed society. In addition, the human behaviours can reflect their real friendships to some extent. We find that the distinct characteristics of engineered versus natural growth processes is evident. Engineered networks are more difficult to mimic through random generative processes and are hence rarer among the space of all networks.

Furthermore, based on the higher-order connectivity patterns, the recently research has developed a nice generalized model for directed networks, especially for clustering networks [31]. More assortativity-related concepts also have been proposed for directed networks [32], using the assortativity modify or construct directed network models should be accepted more attentions in future.

\section{Acknowledgments}

Keke Shang is supported by China Scholarship Council and Michael Small is supported by Australian Research Council Discover Project DP1401100203.

\section{References}

[1] A.-L. Barabási, R. Albert, Emergence of scaling in random networks, science 286 (5439) (1999) 509-512. doi:10.1126/science.286.5439.509. 
[2] F. Papadopoulos, M. Kitsak, M. Á. Serrano, M. Boguná, D. Krioukov, Popularity versus similarity in growing networks, Nature 489 (7417) (2012) 537-540. doi:10.1038/nature11459.

[3] K. Judd, M. Small, T. Stemler, What exactly are the properties of scale-free and other networks?, EPL (Europhysics Letters) 103 (5) (2013) 58004. doi:10.1209/0295-5075/103/ 58004.

[4] M. Small, Y. Li, T. Stemler, K. Judd, Growing optimal scale-free networks via likelihood, Physical Review E 91 (4) (2015) 042801. doi:10.1103/PhysRevE.91.042801.

[5] A. Clauset, C. Moore, M. E. Newman, Hierarchical structure and the prediction of missing links in networks, Nature 453 (7191) (2008) 98-101. doi:10.1038/nature06830.

[6] L. Lü, T. Zhou, Link prediction in complex networks: A survey, Physica A: Statistical Mechanics and its Applications 390 (6) (2011) 1150-1170. doi:10.1016/j.physa.2010.11. 027.

[7] K.-k. Shang, W.-s. Yan, M. Small, Evolving networks-using past structure to predict the future, Physica A: Statistical Mechanics and its Applications 455 (2016) 120-135. doi:10. $1016 / j$.physa. 2016.02 .067$.

[8] Y. Liu, C. Zhao, X. Wang, Q. Huang, X. Zhang, D. Yi, The degree-related clustering coefficient and its application to link prediction, Physica A: Statistical Mechanics and its Applications 454 (2016) 24-33. doi:10.1016/j.physa.2016.02.014.

[9] M. Molloy, B. Reed, A critical point for random graphs with a given degree sequence, Random structures \& algorithms 6 (2-3) (1995) 161-180. doi:10.1002/rsa.3240060204.

[10] M. E. Newman, S. H. Strogatz, D. J. Watts, Random graphs with arbitrary degree distributions and their applications, Physical review E 64 (2) (2001) 026118. doi:10.1103/PhysRevE.64. 026118.

[11] M. Piraveenan, M. Prokopenko, A. Y. Zomaya, Information-cloning of scale-free networks, in: European Conference on Artificial Life, Springer, 2007, pp. 925-935. doi:10.1007/ 978-3-540-74913-4_93. 
[12] D. Kasthurirathna, M. Piraveenan, Cyclic preferential attachment in complex networks, Procedia Computer Science 18 (2013) 2086-2094. doi:10.1016/j.procs.2013.05.378.

[13] M. Piraveenan, M. Prokopenko, A. Y. Zomaya, Local assortativity and growth of internet, The European Physical Journal B 70 (2) (2009) 275-285. doi:http://dx.doi.org/10.1140/ epjb/e2009-00219-y.

[14] R. Xulvi-Brunet, I. M. Sokolov, Changing correlations in networks: assortativity and dissortativity, Acta Physica Polonica B 36 (2005) 1431.

[15] X.-K. Xu, J. Zhang, M. Small, Rich-club connectivity dominates assortativity and transitivity of complex networks, Physical Review E 82 (4) (2010) 046117. doi:10.1103/PhysRevE.82. 046117.

[16] V. Batagelj, A. Mrvar, Pajek datasets, http://vlado.fmf.uni-lj.si/pub/networks/data/ (2006).

[17] D. Lusseau, K. Schneider, O. J. Boisseau, P. Haase, E. Slooten, S. M. Dawson, The bottlenose dolphin community of doubtful sound features a large proportion of long-lasting associations, Behavioral Ecology and Sociobiology 54 (4) (2003) 396-405. doi:10.1007/ s00265-003-0651-y.

[18] R. Mastrandrea, J. Fournet, A. Barrat, Contact patterns in a high school: a comparison between data collected using wearable sensors, contact diaries and friendship surveys, PloS one 10 (9) (2015) e0136497. doi:10.1371/journal.pone.0136497.

[19] Sociopatterns, http://www.sociopatterns.org/, accessed on 8-August-2016.

[20] D. J. Watts, S. H. Strogatz, Collective dynamics of 'small-world' networks, Nature 393 (6684) (1998) 440-442. doi:10.1038/30918.

[21] L. A. Adamic, N. Glance, The political blogosphere and the 2004 us election: divided they blog, in: Proceedings of the 3rd international workshop on Link discovery, ACM, 2005, pp. 36-43. doi:10.1145/1134271.1134277.

[22] M. E. Newman, Finding community structure in networks using the eigenvectors of matrices, Physical review E 74 (3) (2006) 036104. doi:10.1103/PhysRevE.74.036104. 
[23] D. Bu, Y. Zhao, L. Cai, H. Xue, X. Zhu, H. Lu, J. Zhang, S. Sun, L. Ling, N. Zhang, et al., Topological structure analysis of the protein-protein interaction network in budding yeast, Nucleic acids research 31 (9) (2003) 2443-2450. doi:10.1093/nar/gkg340.

[24] L. Isella, J. Stehlé, A. Barrat, C. Cattuto, J.-F. Pinton, W. Van den Broeck, What's in a crowd? analysis of face-to-face behavioral networks, Journal of theoretical biology 271 (1) (2011) 166-180. doi:10.1016/j.jtbi.2010.11.033.

[25] M. E. Newman, Mark newman datasets, http://www-personal.umich.edu/ mejn/netdata/ (2006).

[26] L. Lü, T. Zhou, Link prediction in weighted networks: The role of weak ties, EPL (Europhysics Letters) 89 (1) (2010) 18001. doi:10.1209/0295-5075/89/18001.

[27] J. A. Hanley, B. J. McNeil, The meaning and use of the area under a receiver operating characteristic (roc) curve., Radiology 143 (1) (1982) 29-36. doi:10.1148/radiology.143.1. 7063747.

[28] S. Maslov, K. Sneppen, Specificity and stability in topology of protein networks, Science 296 (5569) (2002) 910-913. doi:10.1126/science.1065103.

[29] M. Small, L. Hou, L. Zhang, Random complex networks, National Science Review 1 (3) (2014) 357-367. doi:10.1093/nsr/nwu021.

[30] K.-k. Shang, X.-k. Xu, Construction and application for null models of complex networks based on randomized algorithms, Journal of University of Electronic Science and Technology of China 43 (2014) 7-20. doi:10.3969/j.issn.1001-0548.2014.01.002.

[31] A. R. Benson, D. F. Gleich, J. Leskovec, Higher-order organization of complex networks, Science 353 (6295) (2016) 163-166. doi:10.1126/science.aad9029.

[32] M. Piraveenan, M. Prokopenko, A. Zomaya, Assortative mixing in directed biological networks, IEEE/ACM Transactions on Computational Biology and Bioinformatics (TCBB) 9 (1) (2012) 66-78. doi:10.1109/TCBB.2010.80.

\section{Appendix}



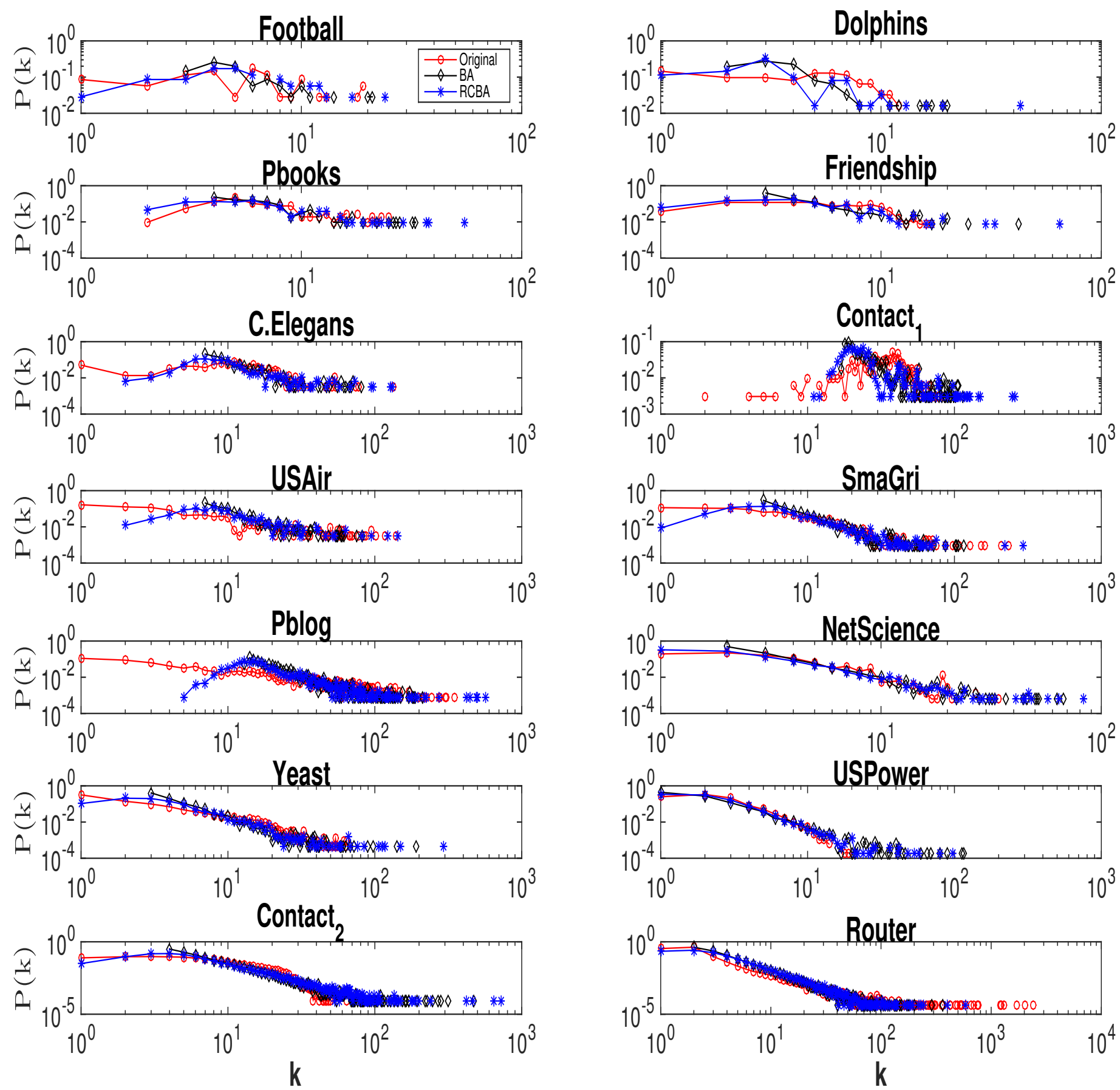

Figure 11: (Color online) The degree distribution of the original real networks, the $B A$ networks and the $R C B A$ networks. 


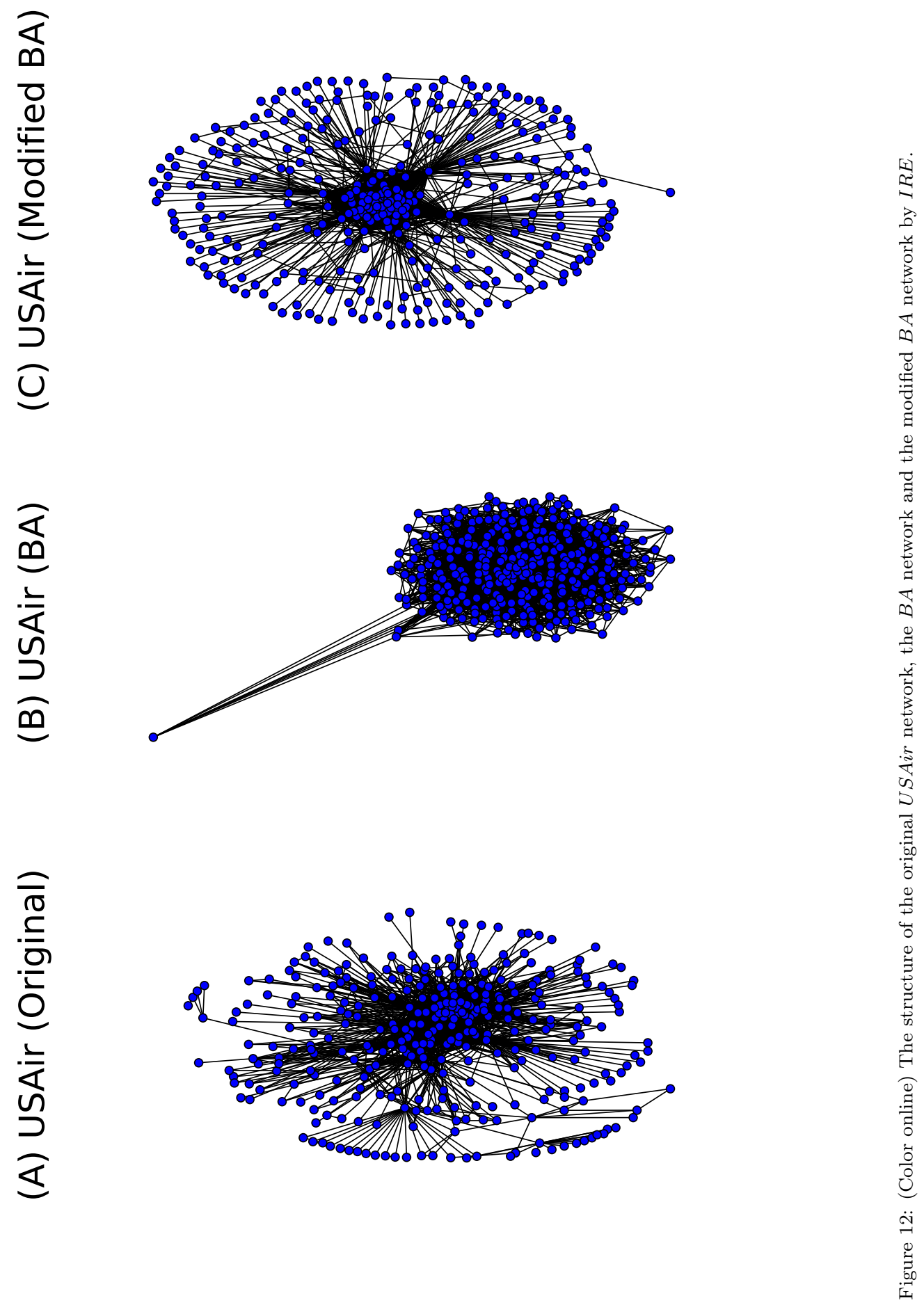

